\title{
Research on the Forming Process of Creative Industrial Cluster Based on the Game Model
}

\author{
Yuhua Li \\ School of Management \\ Harbin University of Science and Technology \\ Harbin, China \\ liyuhua71@163.com
}

\author{
Lulu Yin \\ School of Management \\ Harbin University of Science and Technology \\ Harbin, China \\ yinlulush@126.com
}

\begin{abstract}
The paper applies game theory to study joining behavior of core enterprises and cluster partners during the process of clustering development of creative industry, and it establishes the game model by employing discount factor, income risk coefficient, synergistic coefficient, creative industrial cluster's trust degree and effect coefficient of cooperative output. Based on the game model, this paper analyzes the constraints of forming the creative industrial cluster, and puts forward the strategies to promote the formation of creative industrial cluster.
\end{abstract}

Keywords- game of joining behavior; core enterprises; cluster partners; creative industrial cluster

\section{INTRODUCTION}

As the sunrise industry of 21 century, creative industry has been regarded as a key industry to promote regional economic growth [1]. The creative industry origins from individual creativity, skill and ability, and create wealth and employment through the use of intelligence [2]. It features as innovation, permeability, high added value, strong radiation, high-tech and high-risk [3]. Creative industry penetrates into the traditional industry insufficiently, fails to integrate and extend the industrial chain effectively, and lake of specialized division of labor. The creative industry in China can't reduce costs and obtain scale economy effectively [4]. Therefore, the development of creative industrial cluster is necessary.

During the process of clustering development, it is considered effectively that core enterprises act as the leader and other minor enterprises provide specialized products or services to the core enterprises. With this model, cluster forms gradually as the core enterprises is the dominator, and the small enterprises are looking for the cooperation with them in order to avoid risks and save costs. So, the essence of the forming process of creative industrial cluster is the decision-making evolution process of core enterprises and cluster partners under the combined various factors. The trustful cooperation between core enterprises and cluster partners is important for the formation and stable development of creative industrial cluster. From a game perspective, the forming process of creative industrial cluster is a dynamic game process between core enterprises and cluster partners, which is based on cooperation. Their trustful cooperation is an important driving force for clustering development of creative industry. So, the thesis will

Supported by the National Natural Science Foundation of China (70773032), and by the Humanities and Social Science Research Project of State Education Ministry (10YJC630133), and by the Philosophy and Society Science Significant Decision Consultation Project of Heilongjiang Province (11G004), and by Humanities and Social Science Research Project of Heilongjiang Provincial Department of Education (12522041), and by Young and Top-notch Innovative Talents Training Plan of Harbin University of Science and Technology. research the joining behavior of core enterprises and cluster partners based on a game model.

\section{CONSTRUCTION AND ANALYSIS OF THE COOPERATIVE GAME MODEL}

\section{A. Assumption of the Game Model}

In order to facilitate the analysis, assume that the cooperative game only occurs between a core creative enterprise (firm A) and a cluster partner (firm B).By previous research, the $\mathrm{N}$ times repeated game between core enterprise and cluster partner has the following properties.

1) The original game of repeated game. As the incompletion of information, some indicators are changing with the increasing number of the game. In order to simplify the calculation, regarding the $\mathrm{N}$ times repeated game as the original game.

2) The game has the order. The core enterprise gets the first choice to reach an agreement as its advantages in capital, talent, technology and other aspects. The core enterprise invites the cluster partner to join the cluster by investigating its qualification firstly. And then, the cluster partner decides whether to accept the cooperation by analyzing the risks and expected yields comprehensively.

3) The strategies of the game. There are only two strategies, namely, cooperate and decline, $\mathrm{S}=\left\{\mathrm{s}_{1}, \mathrm{~s}_{2}\right\}=\{\mathrm{c}, \mathrm{d}\}$, and the both sides don' t consider the impact of their decision-making to the other participant. In the Nth time cooperative game, the probability of cooperate for core enterprise is $\mathrm{P}_{\mathrm{A}}$, decline is 1$\mathrm{P}_{\mathrm{A}}$; the probability of cooperate for cluster partner $\mathrm{B}$ is $\mathrm{P}_{\mathrm{B}}$, decline is $1-\mathrm{P}_{\mathrm{B}}$.

4) The yields of the game. Different combinations of strategies will produce different yields, there are four combinations of yields in the game.

$\mathrm{G}=\{[\mathrm{GA}(\mathrm{c}, \mathrm{c}), \mathrm{GB}(\mathrm{c}, \mathrm{c})] ;[\mathrm{GA}(\mathrm{c}, \mathrm{d}), \mathrm{GB}(\mathrm{c}, \mathrm{d})] ;[\mathrm{GA}(\mathrm{d}, \mathrm{c})$, GB $(d, c)]$; [GA (d, d), GB (d, d)] .

\section{B. The Analysis of the Cooperative Game Model}

Introduce the following concepts in order to do the quantitative analysis of the partnership. 
1) Discount factor $\delta$. Assuming that, one company can gain the yields as $I_{1}$ from the first cooperation, gain $I_{2}$ from the second cooperation, ..., the $\mathrm{N}$ times cooperative income as $\mathrm{In}$. the discounted value of $\mathrm{N}$ times cooperation are $\mathrm{I}_{1}, \mathrm{I}_{2} \delta, \ldots, \mathrm{I}_{\mathrm{n}} \delta^{\mathrm{n}-1}$ $(0<\delta<1)$. With the increase of cooperation, the actual income will be reduced when the company gives up the cooperation. The company with small value of $\delta$ will choose to cooperate as soon as possible, or the lost of opportunity cost will be greatly increased. So, we can introduce the discount factor $\delta$ to show the unequal relationship of core enterprise and cluster partner.

2) Income risk coefficient $\eta$. It is blind to forecast the yields only but not concern the risks. Income risk coefficient is an aggregative indicator to measure the various risk factors of the income, the range is $[0,1]$.

3) Synergistic coefficient r. The high efficiency of knowledge spillover is helpful to improve the competitive strength of the cluster, the synergistic coefficient $r(r>0)$ has a positive correlation with this kind of competitive strength. Namely, the higher efficiency of information exchange and sharing in creative industrial cluster, the higher synergistic efficiency of the cluster, otherwise lower.

4) Creative industrial cluster's trust degree $\theta$. The creative industrial cluster's trust is formed by enterprises' repetitious cooperation, presenting in rational increment. In other words, the value of $\theta(\theta>0)$ is increased with the increase of the number of inter-firm cooperation, and will get a positive incentive from each successful cooperation. The larger value of $\theta$, the higher cooperative efficiency and greater gains, so it can be regarded as the positive feedback factor of cooperation.

5) Effect coefficient of cooperative output $\omega$. It's a composite indicator to measure the effectiveness of cooperation, related to the difficulty, technical content and overall profitability of the project. The more likely to cooperate, the lower technical content and higher overall profits will lead a bigger value of $\omega$.

After introduce the above income-related factors, we can make the following assumptions in order to calculate the payoff function [5]:

(1) Assuming that the total investment of firm A and B in the Nth cooperation is I (including the inputs of manpower, material, capital and other elements, and convert the inputs into currency). Calculating their investment funds as joint-stock, the share of firm A is $a$, firm B is $b$, and $a+b=1$.

(2) If the firms both choose to cooperate, they will get the payoff by their input share; If firm A choose to cooperate, firm $\mathrm{B}$ declines the cooperation, the inputs of firm A can be regarded as the gains of firm $\mathrm{B}$, and vice versa; If firm A and B are all decline the cooperation, their payoff are all zero.

(3) The payoff of the gamer should consider the risk coefficient $\eta$, namely, the expected return after risk discount $\mathrm{G}=\eta \times$ payoff-expenditure.

(4) The present value of game parties' expected return. According to the discount factor $\delta$, the present value of expected return is NPV $=\mathrm{G} \times \delta^{\mathrm{n}-1}$, and it is the ultimate basis for decision-making. Due to the unequal relationship, the discount factor of firm $A$ is bigger than $B$, namely $\delta_{A}>\delta_{B}$.

Take core enterprise $\mathrm{A}$ as an example, calculating its strategies and expected returns in the game.

Policy Portfolio one: Core enterprise A and cluster partner $\mathrm{B}$ are all choose to cooperate:

$$
\left.N P V_{\mathrm{A}}(\mathrm{c}, \mathrm{c})=\delta_{A}^{n-1} \times G_{A}(c, c)=\delta_{A}^{n-1}\left[\eta_{A} p_{A} p_{B}{ }^{a \omega \nu} A^{\left(1+\theta_{A}\right.}\right)^{n-1} I-p_{A} p_{B}{ }^{a I}\right]
$$

Namely,

$$
N P V_{G_{A}(c, c)}=\delta_{A}^{n-1} p_{A} p_{B}^{a I}\left[\eta_{A} \omega \gamma_{A}\left(1+\theta_{A}\right)^{n-1}-1\right]
$$

Policy Portfolio two: Firm A chooses to cooperate, firm B declines the cooperation:

$$
N P V_{G_{A}(c, d)}=\delta_{A}^{n-1} \times G_{A}(c, d)=-\delta_{A}^{n-1} p_{A}\left(1-p_{B}\right) a I
$$

Policy Portfolio three: Firm A decline the cooperation and the firm B chooses to cooperate:

$$
N P V_{G_{A}(d, c)}=\delta_{A}^{n-1} \times G_{A}(d, c)=\delta_{A}^{n-1} \eta_{A}\left(1-p_{A}\right) p_{B} b I
$$

Policy Portfolio four: The two sides are all decline the cooperation:

$$
N P V_{\mathrm{G}_{\mathrm{A}}}(d, d)=0
$$

TABle 1 The Payoff Matrix of CoOperative Game Between A AND B

\begin{tabular}{|c|c|c|}
\hline Cooperate & Decline \\
\hline Cooperate & $\begin{array}{c}\delta_{A}^{n-1} p_{A} p_{B}{ }^{a I\left[\eta_{A}{ }^{\omega \gamma}{ }_{A}\left(1+\theta_{A}\right)^{n-1}-1\right]} \\
\delta_{B}^{n-1} p_{A} p_{B} b I\left[\eta_{B} \omega \gamma_{B}\left(1+\theta_{B}\right)^{n-1}-1\right]\end{array}$ & $\begin{array}{c}-\delta_{A}^{n-1} p_{A}\left(1-p_{B}\right) a I, \\
\delta_{B}^{n-1} \eta_{B} p_{A}\left(1-p_{B}\right) a I\end{array}$ \\
\hline Decline & $\delta_{A}^{n-1} \eta_{A}\left(1-p_{A}\right) p_{B} b I$, \\
$-\delta_{B}^{n-1}\left(1-p_{A}\right) p_{B} b I$ & 0,0 \\
\hline
\end{tabular}

\section{ANALYSIS OF THE FORMATION CONDITION FOR CREATIVE INDUSTRIAL CLUSTER}

During the forming process of creative industrial cluster, the decision-making only depends on the numeral value of the expected return after risk discount between cooperate and decline. Take core enterprise $\mathrm{A}$ as an example to explore the strategy selected conditions.

If firm A choose to cooperate, $\triangle N P V_{\mathrm{G}_{\mathrm{A}}}$ must be bigger or equal to zero, it will meet the equation:

$$
\Delta N P V_{G_{A}}=\sum_{i=1}^{4} N P V_{G_{i A}}\left(p_{A}=1\right)-\sum_{i=1}^{4} N P V_{G i A}\left(p_{A}=0\right) \geq 0(i=1,2,3,4)
$$

According to (2), (3), (4), (5) and the known conditions, we have: 


$$
\left\{\begin{array}{l}
\sum_{i=1}^{4} N P V_{G_{i A}}\left(p_{A}=1\right)=\delta_{A}^{n-1} a I\left[\eta_{A} p_{B} \omega \gamma_{A}\left(1+\theta_{A}\right)^{n-1}-1\right] \\
\sum_{i=1}^{4} N P V_{G_{i A}}\left(p_{A}=0\right)=\delta_{A}^{n-1} \eta_{A} p_{B} b I \\
a+b=1
\end{array}\right.
$$

Substitute (7) into (6), have:

$$
a=f\left(\omega, n, \gamma_{A}, \eta_{A}, \theta_{A}, P_{B}\right) \geq \frac{1}{\omega \gamma_{A}\left(1+\theta_{A}\right)^{n-1}+1-\frac{1}{\eta_{A} P_{B}}}
$$

The above equation is the constraint of $\mathrm{A}$ and $\mathrm{B}$ to achieve the cooperation, so $p_{B} \geq 0.5$. Then we can analyze the specific conditions of forming creative industrial cluster.

1) When $n, \gamma_{A}, \eta_{A}, \theta_{A}, p_{B}$ are constant, if $\omega$ is larger, $a$ can be relatively small; in the contrary, if $\omega$ is smaller, $a$ should be relatively large. The first cooperative constraint of core enterprise A is: the higher effect of cooperative output, the higher profit margins will be, namely, the cooperation will bring greater return and it is easy to form even if the input of firm A is small; contrarily, firm A should input a larger share in order to reach a cooperation when the expected return is small.

2) When $\omega, n, \eta_{A}, \theta_{A} p_{B}$ are constant, if $\gamma_{A}$ is larger, $a$ can be relatively small; if $\gamma_{A}$ is smaller, $a$ should be relatively large. The second cooperative constraint of firm A is: if its synergistic capacity is strong, the cooperation can be reached even the input is small; contrarily, more input is needed when the synergistic capacity is poor.

3) When $\omega, \gamma_{A}, \eta_{A}, p_{B}$ are constant, if $n, \theta_{A}$ are larger, $a$ can be relatively small; $a$ must be larger if $n, \theta_{A}$ are smaller. The third cooperative constraint of firm A is: the cooperation can be reached even if firm A inputs less when a trustful relationship is established with the increasing number of cooperation; without trustful relation, firm A can only attract the other side with more investment.

4) When $\omega, n, \gamma_{A}, \theta_{A}, p_{B}$ are constant, if $\eta_{A}$ is larger, $a$ can be relatively small; $a$ must be larger as $\eta_{A}$ is smaller. The fourth constraint for firm A is: cooperation can be reached even if firm A invests less when the risk is higher; contrarily, with the less risk, firm A must input more.

5) When $\omega, n, \gamma_{A}, \eta_{A}, \theta_{A}$ are constant, if $p_{B}$ is larger, $a$ can be relatively small; $a$ must be larger as $p_{B}$ is smaller. The fifth constraint for firm A is: One party's willing to cooperate will be a great incentive to the other party. For firm $\mathrm{A}$, if firm B has an intense desire to cooperate, the cooperation can be reached even if the investment of firm A is small; contrarily, firm A can only increase the investment.

The above analyses describe the strategies of core enterprise A, firm B as the same. So, if firm B choose to cooperate in Nth game, it must meet the following condition:

$$
b=f\left(\omega, n, \gamma_{B}, \eta_{B}, \theta_{B}, P_{A}\right) \geq \frac{1}{\omega \gamma_{B}\left(1+\theta_{B}\right)^{n-1}+1-\frac{1}{\eta_{B} P_{A}}}
$$

The cooperative constraints for both core enterprise $A$ and cluster partner B are:

$\left\{\begin{array}{l}a=f\left(\omega, n, \gamma_{A}, \eta_{A}, \theta_{A}, P_{B}\right) \geq \frac{1}{\omega \gamma_{A}\left(1+\theta_{A}\right)^{n-1}+1-\frac{1}{\eta_{A} P_{B}}} \\ b=f\left(\omega, n, \gamma_{B}, \eta_{B}, \theta_{B}, P_{A}\right) \geq \frac{1}{\omega \gamma_{B}\left(1+\theta_{B}\right)^{n-1}+1-\frac{1}{\eta_{B} P_{A}}} \\ a+b=1\end{array}\right.$

In order to simplify the calculation, let

$\frac{1}{\omega \gamma_{A}\left(1+\theta_{A}\right)^{n-1}+1-\frac{1}{\eta_{A} P_{B}}}=\sigma_{A}, \frac{1}{\omega \gamma_{B}\left(1+\theta_{B}\right)^{n-1}+1-\frac{1}{\eta_{B} P_{A}}}=\sigma_{B}$,
so $a \geq \sigma_{A}, b \geq \sigma_{B}$.

The feasible region for firm $A$ and $B$ in the Nth game are described in figure 1 based on (10):

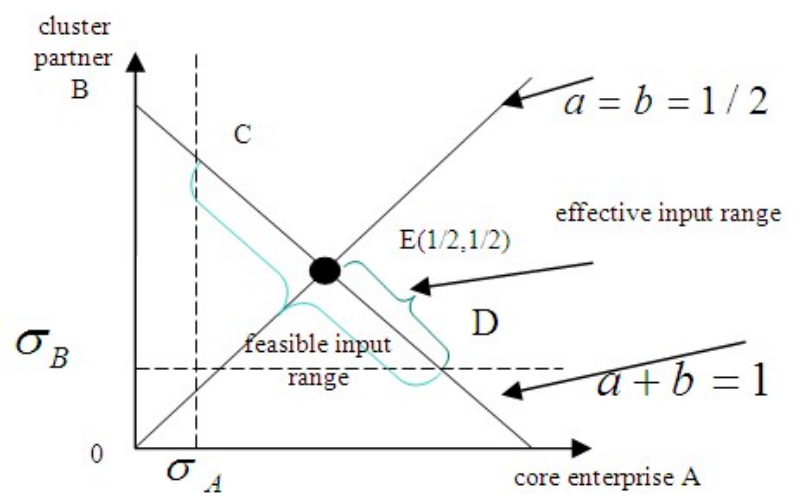

Figure 1 the effective input range of cooperation between core enterprise A and cluster partner $B$

In figure $1,[C, D]$ is the feasible region for firm $A$ and $B$ both choose to cooperate, and reach an equilibrium at the point $\mathrm{E}(1 / 2,1 / 2)$. As firm $\mathrm{A}$ is in the core position, the competitive advantage of firm $\mathrm{A}$ is larger than firm B. Namely, synergistic coefficient $r$ and trust degree $\theta$ of firm $A$ are both larger than those of firm B, as $\gamma_{A}>\gamma_{B}, \theta_{A}>\theta_{B}$; Additionally, firm B is more eager to join the cluster because of the needs of costs cutting and resource optimal allocation, so the joining probability of $\mathrm{B}$ is larger than $\mathrm{A}$, as $p_{B}>p_{A}$. Finally, the risk of firm $A$ is bigger than $B$ because of its more input, as $\eta_{A}>\eta_{B}$. It follows that $\sigma_{A} \leq \sigma_{B}$, the minimum input of firm $B$ is greater than firm A. In other words, the cooperative barriers of firm $B$ will be higher than firm $A$, and for the restrictions of the capital and technology, it is more difficult for firm B to join the cluster. To achieve cooperation this time, the core enterprise A must remedy or make concessions, which can be regarded as increasing financial and technical inputs or 
promoting the profit margins of firm B. As a result, the investment of firm $\mathrm{A}$ is greater than firm $\mathrm{B}$, the valid input range is $[\mathrm{E}, \mathrm{D}]$, and the more close to point $\mathrm{D}$, the more possibility to reach cooperation.

\section{The Strategies Analysis to Promote the FORMATION OF CREATIVE INDUSTRIAL CLUSTER}

Based on the above analyses of cooperative game between core enterprise and cluster partner, the promoting strategies for the formation of creative industrial cluster are as follows:

1) Improving the effect coefficient of cooperative output. Promoting the output efficiency is benefit for the clustering development of creative industry. The promotion measures are as follows: firstly, integrating elements as resource, talent and technology based on industrial chain; secondly, cultivating and introducing creative talents to bring innovation; finally, strengthen guidance and control of cooperation, communication and feedback of problems timely.

2) Promoting knowledge spillovers and enhancing the synergistic coefficient. Firstly, improving culture collaboration, organizing discussing regularly to form a mutual communication atmosphere; secondly, building the public information platform to facilitate information access, exchange and sharing. Lastly, setting up guiding mechanism to control the expansion of those low resource utilization firms, curb excessive competition to enhance knowledge flow and technical transfer.

3) Increasing the number of cooperation and promoting trust level, which are positively correlated factors. The increased number of cooperation can enhance the mutual understanding and trust which are benefit to establish a longterm and stable cooperative relation.
4) Improving input ratio. The creative industrial clusters are often take axis-wheeled pattern which regards core enterprise as the leader. Increase the input of core enterprise and other enterprises supporting its production to form "upstream development, midstream expansion and downstream extension" industrial chain. The core enterprise transfers some profits to small firms through this pattern.

5) Improving the cooperative probability. The cooperative probability is directly related to potential cooperative interests, industrial associate degree, mutual complementary of assets, cooperative culture in cluster, social transmission mechanism and corporate trustworthiness [6]. The asymmetric information leads to high costs on formation and conduction contracts. Relation contract and "retaliation mechanism of trigger strategy" can effectively curb opportunistic behavior after contracting and improve the credit of enterprises to fulfill their contracts, thus to promote the cooperative efficiency of the entire cluster.

\section{REFERENCES}

[1] S. F. Han, X. M. Li, "Innovation, culture and the analysis of creative industry value". Studies in Science of Science, vol. S2, pp. 555-560, 2008.

[2] GB Department, GB Department of Culture Media and Sport. GB, Mapping Document, 1998.

[3] W. W. Li, Introduction to Creative Industries. Shanghai, Xuelin Publisher, 2007.

[4] H. Xing, "Research on the Value-chain Merge and the Development Path of the Cultural and Creative Industry". Economic Management Journal, vol. 2, pp. 37-41, 2009.

[5] Y. J. Liu, J. P. Yang, "Game Analysis on Synergic Competition Innovation Behavior of Enterprises in Cluster". System and Engineering, vol. 6, pp. 22-26, 2002.

[6] C. Antonelli, "Collective Knowledge Communication and Innovation: The Evidence of Technology Districts". Regional Studies, vol. 6, pp. 535-547, 2000. 